\title{
Autochthone Gebirgswälder in der Schweiz anhand von baumbewohnenden Flechten erkennen
}

\author{
Lyudmyla Dymytrova \\ Urs-Beat Brändli \\ Silvia Stofer \\ Christoph Scheidegger
}

\author{
Eidgenössische Forschungsanstalt für Wald, Schnee und Landschaft $(\mathrm{CH})$ \\ Eidgenössische Forschungsanstalt für Wald, Schnee und Landschaft $(\mathrm{CH})$ \\ Eidgenössische Forschungsanstalt für Wald, Schnee und Landschaft $(\mathrm{CH})$ \\ Eidgenössische Forschungsanstalt für Wald, Schnee und Landschaft $(\mathrm{CH})^{*}$
}

\section{Autochthone Gebirgswälder in der Schweiz anhand von baumbewohnenden Flechten erkennen}

\begin{abstract}
Alte ungestörte Waldbestände mit einer langen Waldgeschichte sind für den Natur- und Artenschutz von besonderem Interesse. Häufig werden baumbewohnende Flechten als Indikatoren für solche autochthonen Bestände verwendet. In der vorliegenden Studie testeten wir, inwiefern sich 14 baumbewohnende Flechtenarten, die von Forstpraktikern und Forstpraktikerinnen einfach anzusprechen sind und die über ein geringes Ausbreitungspotenzial verfügen, spezifische Anforderungen an Kleinstandorte stellen und oft auf alte Bäume angewiesen sind, sich in den Schweizer Gebirgswäldern als Indikatorarten für autochthone Waldbestände eignen würden. Als autochthon betrachteten wir dabei Waldbestände mit einem naturnahen Nadelholzanteil, einem Mindestalter von 90 Jahren, einer kontinuierlichen Waldbedeckung während 120 Jahren und ohne Hinweise auf einen historischen flächenhaften Eingriff wie Kahlschlag oder Aufforstung. Für die Studie haben die regulären Feldteams des Schweizerischen Landesforstinventars (LFI) im Rahmen der dritten Erhebung (2004-2006) auf sämtlichen Probeflächen im 1,4-km-Netz an jeweils etwa drei Bäumen die potenziellen Indikatorarten aufgenommen. Ausgewertet wurden sodann die Daten für die 3510 im Gebirgswald liegenden Probeflächen unter Verwendung der LFI-Daten zur Waldstruktur, Waldgeschichte und Waldbewirtschaftung. Insgesamt war die Anzahl Indikatorarten in autochthonen Waldbeständen signifikant höher als in nicht autochthonen, wobei sie mit der Höhenlage und der biogeografischen Region erheblich variierte. Unsere Untersuchungen zeigten, dass Strauchflechten der Gattungen der Moosbärte (Bryoria sp. div.) und der Bartflechten (Usnea sp. div.) wie auch die Sparrige Pflaumenflechte (Evernia divaricata) und die Wolfsflechte (Letharia vulpina), Blattflechten wie die Oliven-Schüsselflechte (Cetrelia olivetorum) und die Echte Lungenflechte (Lobaria pulmonaria) sowie Krustenflechten wie die Weissfleckenflechten (Lecanactis abietina und Microcalicium disseminatum), die Stecknadelflechten (Calicium sp. div. und Chaenotheca sp. div.) und die auffällige Borken-Schwefelflechte (Chrysothrix candelaris) zuverlässige Indikatoren für autochthone Waldbestände in Schweizer Gebirgswäldern sind, insbesondere wenn mehrere Indikatorarten gemeinsam vorkommen. Die getesteten Flechten eignen sich deshalb in der Praxis, um auf einfache Weise autochthone Waldbestände beispielsweise für die Ausscheidung von Waldreservaten oder Altholzinseln zu erkennen. Solche Bestände sind für die Förderung der Biodiversität in einer seit Jahrhunderten bewirtschafteten Waldlandschaft von hoher Bedeutung.
\end{abstract}

Keywords: indicator species, epiphytic lichens, old-growth forest, forest continuity, long-term persistent habitats, Swiss National Forest Inventory

doi: 10.3188/szf.2019.0258

* Zürcherstrasse 111, CH-8903 Birmensdorf, E-Mail christoph.scheidegger@wsl.ch

$\mathrm{V}$ iele seltene und gefährdete Arten verschiedener Organismengruppen sind auf autochthone, das heisst alte ungestörte Waldbestände auf Standorten mit langer Waldgeschichte angewiesen (Brin et al 2016, Ellis 2012, Graae \& Sunde 2000, Graham et al 2006, Scheidegger \& Stofer 2015, Wirth 2002). Oftmals zählen diese Arten gar zu den insgesamt 1582 national prioritären Waldarten, also Waldarten, für die die Schweiz in- ternational eine besondere Verantwortung hat und die national gefährdet sind (BAFU 2011, Imesch et al 2015). Für eine effektive Förderung der Biodiversität im Wald sind daher geeignete Ansätze erforderlich, um autochthone Waldbestände zu erkennen und langfristig zu erhalten.

In verschiedenen Regionen der Welt werden baumbewohnende Flechtenarten als Indikatoren für autochthone Waldbestände eingesetzt (Arsenault \& 
Goward 2016, Boch et al 2013, Coppins \& Coppins 2002, Frey 1958, Fritz et al 2008, Josefsson et al 2005, Marmor et al 2011, McCune 2000, Rose 1976, Wirth 1976). Dies ist möglich, weil sich diese Arten einerseits nur auf kurze Entfernung durch vegetative Ausbreitungseinheiten vermehren können und sie andererseits an spezifische Strukturen gebunden sind, die hauptsächlich in alten Waldbeständen vorkommen. Die Flechten-Indikatorarten sind damit auf das Ausbleiben von flächigen Störungen bzw. auf Bestandeskontinuität über längere Zeiträume angewiesen. In buchendominierten Wäldern muss die Dauer der Kontinuität mindestens 350 bis 400 Jahre (Fritz et al 2008) und in Nadelwäldern etwa 200 bis 350 Jahre (Gauslaa \& Ohlson 1997, Marmor et al 2011, Tibell 1992) betragen. Bestände mit solch langer Bestandeskontinuität sind in der Schweiz sehr selten. Denn aufgrund der intensiven Nutzung der Wälder im 18. und 19. Jahrhundert sind nur $0.4 \%$ der Wälder älter als 250 Jahre (Brändli et al 2015). Am häufigsten werden autochthone Bestände in den Gebirgswäldern erwartet.

Bislang mussten in der Schweiz Informationen, um die Bestandesautochthonie erkennen und bewerten zu können, aus Bestandeskarten und Wirtschaftsplänen (Alter, Entstehungsart, bisherige Bewirtschaftung) und durch Befragung der Revierförster und Revierförsterinnen aufwendig zusammengetragen werden, und oftmals blieben die Informationen lückig. Auch der Vergleich von historischen und aktuellen Karten, zu dem Ginzler et al (2011) ein effektives und praktikables Verfahren entwickelt hatten, vermochte nur die Frage der Waldgeschichte («Waldkontinuität») als einen Teilaspekt von Autochthonie zu klären. Daher wurde die Gelegenheit des dritten Landesforstinventars (LFI3) genutzt, um im Rahmen

\begin{tabular}{|l|l|}
\hline Deutscher Name & Wissenschaftlicher Name \\
\hline Bartflechten & Usnea sp. div. \\
\hline Baumbart & Alectoria sarmentosa \\
\hline Bewimperte Schüsselflechten & Parmotrema sp. div. \\
\hline Bleiflechten & Ochrolechia sp. div. \\
\hline Borken-Schwefelflechte & Chrysothrix candelaris \\
\hline Echte Lungenflechte & Lobaria pulmonaria \\
\hline Löcherflechte & Menegazzia terebrata \\
\hline Moosbärte & Bryoria sp. div. \\
\hline Oliven-Schüsselflechte & Cetrelia olivetorum \\
\hline Pockenflechte & Thelotrema lepadinum \\
\hline Sparrige Pflaumenflechte & Evernia divaricata \\
\hline Stecknadelflechten & Calicium sp. div., Chaenotheca sp. div. \\
\hline Weissfleckenflechten & Arthonia byssacea, Bactrospora dryina, \\
\hline Lecanactis abietina, Microcalicium \\
\hline disseminatum \\
\hline
\end{tabular}

Tab 1 Deutsche und wissenschaftliche Namen der 14 getesteten Flechtenarten und -artengruppen. der regulären Felderhebungen in den Jahren 2004 bis 2006 von den Feldteams eine Auswahl von gut kenntlichen baumbewohnenden Flechten aufnehmen zu lassen. Damit sollte geklärt werden, ob sich auch in der Schweiz Indikatorflechten zur Beurteilung der Autochthonie von Waldbeständen eignen.

\section{Material und Methoden}

Insgesamt wurden 14 baumbewohnende Flechtenarten oder -artengruppen (Tabelle 1) als potenzielle Indikatoren für autochthone Waldbestände in der Schweiz getestet. Ausgewählt wurden Arten, die von Forstpraktikern und -praktikerinnen im Feld leicht zu erkennen sind und die sich entweder in anderen Regionen Europas als gute Indikatoren erwiesen haben (Coppins \& Coppins 2002, Fritz et al 2008) oder für die wir eine gute Eignung für die Schweiz vermuteten.

Die Flechtenerhebung wurde während der dritten LFI-Erhebung (2004-2006) auf den kreisförmigen Probeflächen von $500 \mathrm{~m}^{2}$ im 1.4-km-Netz durchgeführt, auf denen alle Bäume mit einem Brusthöhendurchmesser ab $36 \mathrm{~cm}$ vermessen und beurteilt werden. Insgesamt sind 6462 Probeflächen aufgenommen worden, davon 3510 im Gebirgswald, das heisst im Wald der oberen montanen und der subalpinen Stufe. Die Erhebung wurde von den regulären zwölf Feldteams durchgeführt. Vorgängig waren sie an einem eintägigen Einführungskurs in der Beobachtung und Identifizierung der ausgewählten Flechten im Feld geschult worden. Die Einführung basierte auf dem LFI-Handbuch (Scheidegger \& Stofer 2003, Keller 2011). Das Handbuch enthält eine kurze Beschreibung der morphologischen Merkmale und Fotos der Flechten. Jedem Feldteam wurde ausserdem ein Set von Vergleichsproben zur Verfügung gestellt, um die Ansprache der Flechten im Feld überprüfen zu können.

Als Weissfleckenflechten wurden ursprünglich die zwei Arten Arthonia byssacea und Bactrospora dryina, die im Tiefland an alte Eichen gebunden sind, ausgewählt. Die meisten Beobachtungen zu dieser Art stammen jedoch von Gebirgswäldern, was darauf schliessen lässt, dass die Feldteams weitere Arten wie Lecanactis abietina und die nicht lichenisierte Microcalicium disseminatum unter diesem Namen erfasst haben.

Die Flechten wurden innerhalb des $0^{\circ}-90^{\circ}$-Sektors der Probefläche an durchschnittlich drei stehenden lebenden oder toten Probebäumen vom Boden bis auf eine Höhe von $2 \mathrm{~m}$ mit einer Lupe erhoben. Für die Erhebung der Flechten standen für eine Probefläche jeweils zwei Minuten zur Verfügung. Die Häufigkeit jeder Indikatorflechte pro Probefläche wurde mittels Zusammenzählen der Vorkommen pro Baum ermittelt. 

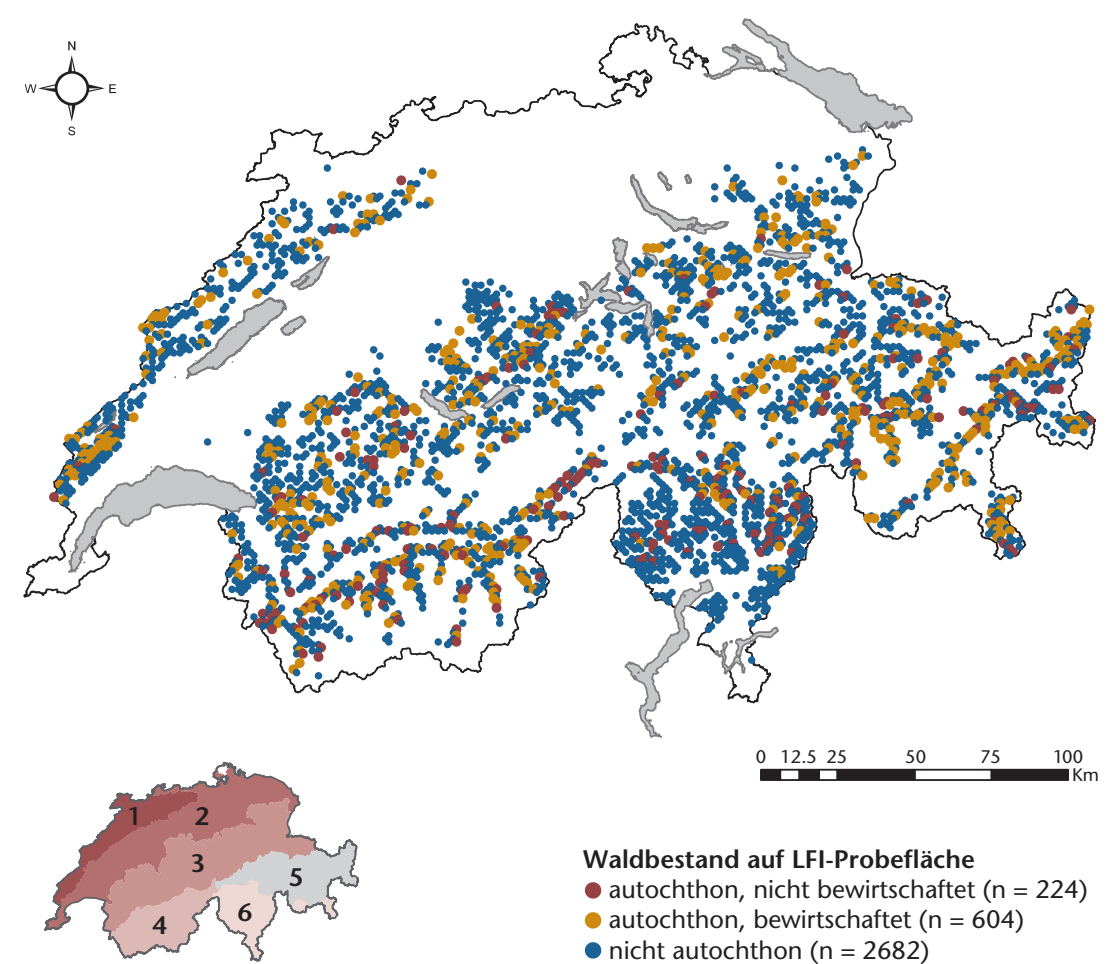

$0 \quad 12.5 \quad 25$
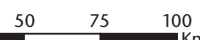

Abb 1 Autochthonie und Bewirtschaftung der Waldbestände auf den LFI-Probeflächen im Schweizer Gebirgswald. Die kleine Karte zeigt die biogeografischen Regionen in der Schweiz: 1: Jura, 2: Mittelland, 3: Nordalpen, 4: Westliche Zentralalpen, 5: Östliche Zentralalpen, 6: Südalpen.

Die Bestandesautochthonie wurde in zwei Schritten bewertet. Zunächst wurde die Kontinuität des Waldes für jede LFI-Probefläche anhand von historischen Karten der Schweiz aus dem Jahr 1880 («Siegfriedkarten») beurteilt (Ginzler et al 2011). In einem zweiten Schritt wurde mittels LFI-Daten geprüft, ob die aktuelle Bestockung die Kriterien für Autochthonie erfüllt. LFI-Waldprobeflächen, die sich 1880 entweder im Wald oder maximal 50 m von

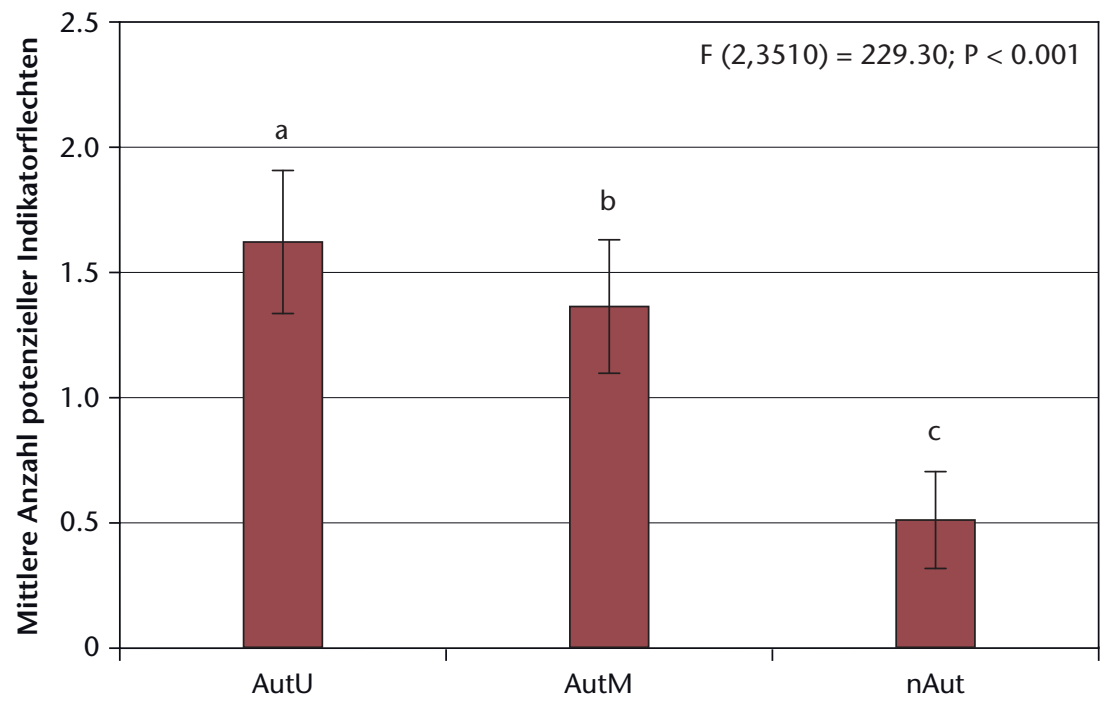

Abb 2 Mittlere Anzahl potenzieller Indikatorflechten in autochthonen, nicht bewirtschafteten (AutU; Mittelwert $=1.62 \pm 1.46$ ), autochthonen, bewirtschafteten (AutM; Mittelwert $=1.36 \pm 1.36)$ und nicht autochthonen (nAut; Mittelwert $=0.51 \pm 0.99)$ Waldbeständen im Schweizer Gebirgswald. Mittelwerte ohne gemeinsame Buchstaben unterscheiden sich signifikant bei $P<0.05$. Abbildung nach Dymytrova et al (2018) einem Waldrand entfernt befanden bzw. eine Einzelbaumsignatur aufwiesen, wurden als autochthon für mindestens 120 Jahre eingestuft, unter der Voraussetzung, dass die aktuelle Bestockung einen naturnahen Nadelholzanteil aufwies, mindestens 90 Jahre alt war, sich nicht aus einer Pflanzung entwickelt hatte und keine Anzeichen von Kahlschlag und Aufforstung zeigte. Die LFI-Probeflächen im Gebirgswald, die die genannten Kriterien erfüllten, wurden als autochthon angesehen $(n=828)$, alle anderen als nicht autochthon ( $\mathrm{n}=2682$; Abbildung 1).

Die Waldbewirtschaftung in der Schweiz weist grosse regionale Unterschiede auf. Generell beträgt in der Schweiz ein normaler Zyklus für waldbauliche Behandlungen (z.B. Pflege, Durchforstung) 5-10 Jahre in Tieflagen, 10-20 Jahre in Gebirgswäldern und auf Standorten mit der niedrigsten Produktivität, z.B. in subalpinen Wäldern bis zur Waldgrenze, 20-50 Jahre (Duc et al 2010). Nach der Definition des LFI gelten alle Waldbestände ohne waldbaulichen Eingriff seit mehr als 50 Jahren als nicht bewirtschaftet. In unserer Studie klassifizierten wir autochthone Waldbestände in zwei Gruppen: «bewirtschaftet», wenn der letzte Eingriff während der letzten 50 Jahre stattfand, und «nicht bewirtschaftet», wenn er vor über 50 Jahren erfolgte.

\section{Statistische Analysen}

Alle Analysen auf der Ebene der Probeflächen wurden mit dem Artdatensatz (3510 Probeflächen $\times$ 14 potenzielle Indikatorarten) durchgeführt. Der Artdatensatz stellt eine Anwesenheits-/Abwesenheitsmatrix der ausgewählten Flechten für die untersuchten Probeflächen dar. Eine Zweiweg-ANOVA mit Post-hoc-Tukey-HSD-Test wurde durchgeführt, um die Auswirkungen von Bestandesautochthonie, Höhe und biogeografischer Region auf die Anzahl der potenziellen Indikatorflechten zu untersuchen.

Die Auswirkungen der Bestandesautochthonie auf die potenziellen Indikatorarten wurden mit den ursprünglichen Häufigkeitsdaten durchgeführt (Dufrêne \& Legendre 1997). Für jede Art wurde der Indikatorwert (IndVal) mit dem Paket «indispecies» (Cáceres 2013) berechnet und anschliessend mit 10000 Monte-Carlo-Permutationen getestet. Weiter wurde die Spezifität jeder potenziellen Indikatorart als durchschnittliche Häufigkeit der Art in der Zielgruppe (autochthon-nicht bewirtschaftet, autochthon-bewirtschaftet, autochthon-unabhängig von der Bewirtschaftung) berechnet (Caro 2010). Der Artikel basiert auf einer grösseren Studie, die von Dymytrova et al (2018) publiziert wurde.

\section{Resultate}

Auf 36\% oder 1249 der im Gebirgswald liegenden Probeflächen wurden potenzielle Indikator- 


\begin{tabular}{|l|r|r|r|r|}
\hline Variationsquelle & \multicolumn{1}{|c|}{ df } & \multicolumn{1}{|c|}{ MS } & \multicolumn{1}{|c|}{ F } & P-Wert \\
\hline Bestandesautochthonie & 2 & 272.3 & 263.54 & $<0.001$ \\
\hline Höhenlage & 1 & 271.0 & 262.24 & $<0.001$ \\
\hline Biogeografische Region & 5 & 16.14 & 15.61 & $<0.001$ \\
\hline $\begin{array}{l}\text { Bestandesautochthonie } \times \text { Höhenlage } \\
\text { Bestandesautochthonie } \times \text { biogeo- }\end{array}$ & 2 & 38.7 & 36.84 & $<0.001$ \\
\hline grafische Region & 9 & 3.2 & 3.12 & $<0.001$ \\
\hline $\begin{array}{l}\text { Höhenlage } \times \text { biogeografische Region } \\
\text { Bestandesautochthonie } \times \text { Höhenlage } \times \\
\text { biogeografische Region }\end{array}$ & 5 & 6.8 & 6.6 & $<0.001$ \\
\hline \begin{tabular}{l} 
Fehler \\
\hline
\end{tabular} & 3476 & 1.0 & 3.4 & 0.004 \\
\hline
\end{tabular}

Tab 2 Die Auswirkungen von Bestandesautochthonie, Höhenlage und biogeografischer Region auf die Anzahl potenzieller Indikatorflechten pro Probefläche.

flechten gefunden. Die durchschnittliche Anzahl pro Probefläche betrug $0.72 \pm 0.02$, die maximale Anzahl 7. Auf $38 \%$ der Probeflächen mit potenziellen Indikatorflechten wurde nur eine dieser Arten beobachtet.

Die Anzahl potenzieller Indikatorflechten war in autochthonen Beständen signifikant höher als in nicht autochthonen. In autochthonen, nicht bewirtschafteten Beständen war sie zudem signifikant höher als in autochthonen, bewirtschafteten (TukeyHSD-Test; P <0.05; Abbildung 2). Nachweisbar ist auch der Einfluss der Höhenlage und der biogeografischen Region (Tabelle 2). So wiesen Probeflächen im Jura und in den Südalpen deutlich weniger po- tenzielle Indikatorarten auf als solche in den Nordund Zentralalpen, und Gebirgswälder wiesen mehr potenzielle Indikatorarten auf als Tieflagenwälder (nicht dargestellt; mehr dazu in Dymytrova et al 2018).

Insgesamt zeigten 9 der untersuchten 14 Flechtenarten eine signifikante Bindung an autochthone Waldbestände (Tabelle 3; P mind. <0.5). Fotos dieser neun Arten finden sich in Abbildung 3. Die Echte Lungenflechte und die Weissfleckenflechten wiesen dabei eine signifikante Bindung an autochthone, nicht bewirtschaftete Waldbestände auf. Mit den Moosbärten, der Borken-Schwefelflechte, der Oliven-Schüsselflechte, der Sparrigen Pflaumenflechte, der Wolfsflechte, den Bartflechten und den Stecknadelflechten sind sieben weitere Flechtenarten mit autochthonen Waldbeständen assoziiert, unabhängig davon, ob die Bestände als bewirtschaftet oder nicht bewirtschaftet gelten (Tabelle 3).

Die Vorkommen der Indikatorarten waren nicht ausschliesslich auf autochthone Waldbestände beschränkt, sondern sie waren auch in nicht autochthonen anzutreffen, hier allerdings mit sehr niedriger relativer Häufigkeit (Tabelle 3). Fünf getestete Flechtenarten - der Baumbart, die Löcherflechte, die Bleiflechten sowie die bewimperten Schüsselflechten und die Pockenflechte - zeigten keine signifikante Bindung an autochthone Waldbestände.

\begin{tabular}{|c|c|c|c|c|c|c|}
\hline Flechtenart & $\begin{array}{c}\text { Spezifität } \\
(\%)\end{array}$ & AutU & AutM & nAut & IndVal & P-Wert \\
\hline Anzahl Probeflächen & & 224 & 604 & 2682 & & \\
\hline \multicolumn{7}{|c|}{ Indikatorarten mit starker ökologischer Bindung an autochthone, nicht bewirtschaftete Bestände } \\
\hline Baumbart & 44.8 & 0.009 & 0.005 & 0.003 & 0.063 & n.s. \\
\hline Echte Lungenflechte & 70.3 & 0.013 & 0.005 & 0.004 & 0.097 & * \\
\hline Weissfleckenflechten & 80.0 & 0.009 & 0 & 0.001 & 0.084 & * \\
\hline Löcherflechte & 49.1 & 0.027 & 0.013 & 0.010 & 0.115 & n.s. \\
\hline \multicolumn{7}{|c|}{ Indikatorarten mit starker ökologischer Bindung an autochthone, bewirtschaftete Bestände } \\
\hline Bleiflechten & 85.6 & 0 & 0.007 & 0.001 & 0.075 & n.s. \\
\hline \multicolumn{7}{|c|}{ Indikatorarten mit starker ökologischer Bindung an autochthone Bestände (ungeachtet der Bewirtschaftung) } \\
\hline Moosbärte & 88.9 & 0.281 & 0.224 & 0.066 & 0.461 & *** \\
\hline Stecknadelflechten & 87.5 & 0.210 & 0.169 & 0.060 & 0.397 & $* * *$ \\
\hline Oliven-Schüsselflechte & 86.0 & 0.049 & 0.060 & 0.023 & 0.221 & $* * *$ \\
\hline Borken-Schwefelflechte & 85.7 & 0.045 & 0.063 & 0.025 & 0.223 & $* * *$ \\
\hline Sparrige Pflaumenflechte & 87.8 & 0.192 & 0.190 & 0.068 & 0.409 & $* * *$ \\
\hline Wolfsflechte & 90.4 & 0.179 & 0.099 & 0.035 & 0.330 & $* * *$ \\
\hline Bartflechten & 86.1 & 0.585 & 0.497 & 0.19 & 0.669 & $* * *$ \\
\hline \multicolumn{7}{|c|}{ Flechtenarten ohne signifikante ökologische Bindung an autochthone Gebirgswälder } \\
\hline Bewimperte Schüsselflechten & n.a. & 0.004 & 0.007 & 0.004 & 0.069 & n.s. \\
\hline Pockenflechte & n.a. & 0.009 & 0.018 & 0.013 & 0.115 & n.s. \\
\hline
\end{tabular}

Tab 3 Indikatorwerte (IndVal), Spezifität (\%) und Häufigkeit von baumbewohnenden Flechten, untersucht auf Parzellen in autochthonen, nicht bewirtschafteten (AutU), autochthonen, bewirtschafteten (AutM) und nicht autochthonen Waldbeständen (nAut) im Schweizer Gebirgswald. Spezifität ist nicht anwendbar (n.a.) für Arten, die keiner Gruppe angehören. Signifikanzniveaus sind ***: $P<0.001,{ }^{* *}: P<0.01,{ }^{*}: P<0.05$, n.s.: nicht signifikante Assoziation. 

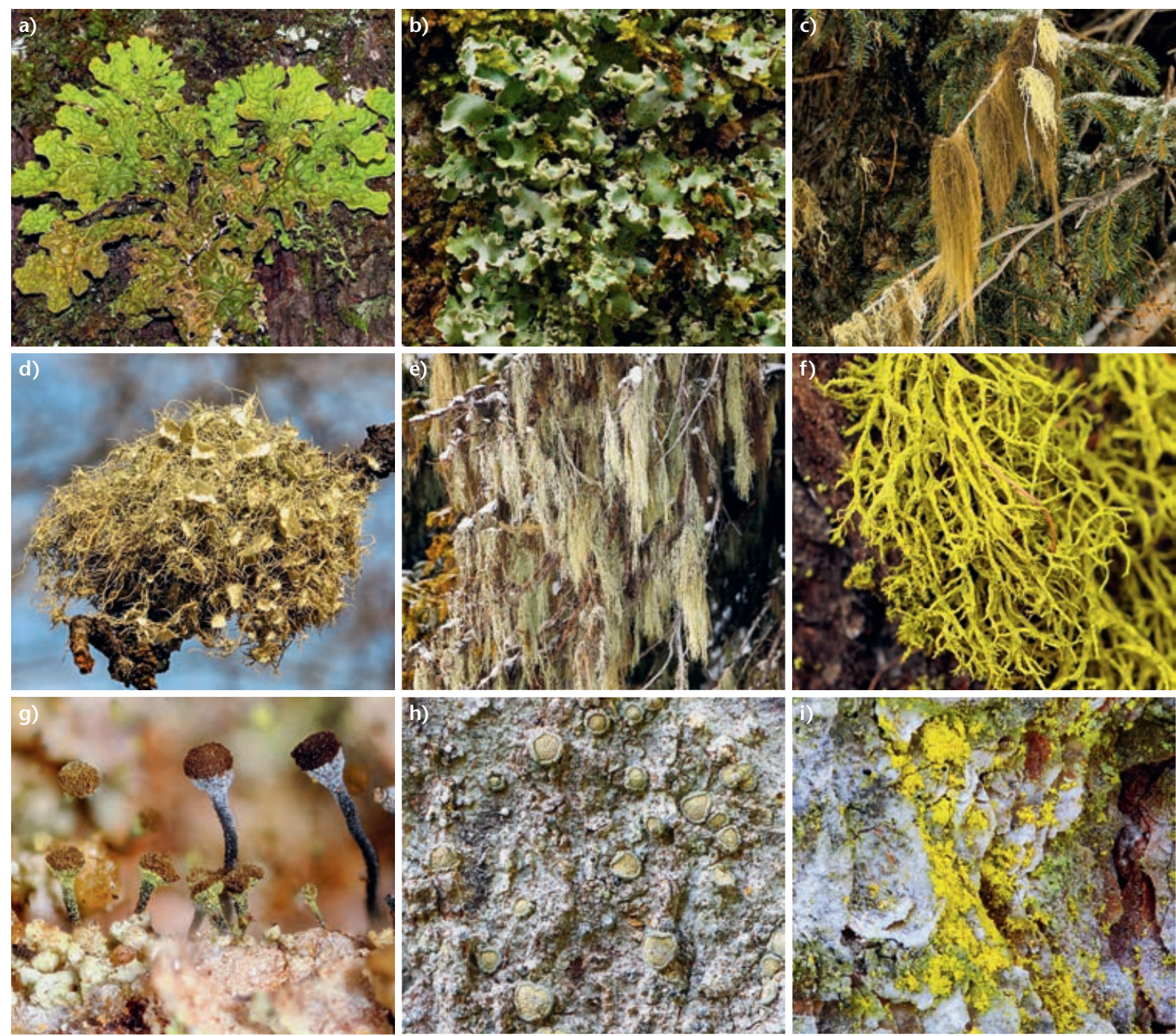

Abb 3 Flechtenarten mit signifikanter Bindung an autochthone Gebirgswälder in der Schweiz. a) Echte Lungenflechte (Lobaria pulmonaria), b) Oliven-Schüsselflechte (Cetrelia olivetorum), c) Moosbärte (Bryoria sp.), d) Bartflechten (Usnea intermedia), e) Sparrige Pflaumenflechte (Evernia divaricata), f) Wolfsflechte (Letharia vulpina), g) Stecknadelflechten (Chaenotheca trichialis und C. chlorella), h) Weissfleckenflechten (Lecanactis abietina), i) Borken-Schwefelflechte (Chrysothrix candelaris).

\section{Diskussion}

Indikatoren für autochthone Bestände in Schweizer Gebirgswäldern

Insgesamt wurde für 9 der 14 untersuchten Flechtenarten eine signifikante Bindung an autochthone Bestände im Schweizer Gebirgswald festgestellt. Sie dürfen als verlässliche Indikatoren betrachtet werden. Denn für viele dieser Flechtenarten wurde dieses Potenzial bereits in anderen Studien nachgewiesen, zum Beispiel für die Borken-Schwefelflechte, die Oliven-Schüsselflechte, die Sparrige Pflaumenflechte sowie für einzelne Arten der Gattung der Bartflechten wie Usnea ceratina und U. florida (Coppins \& Coppins 2002, Fritz et al 2008, Gauslaa 1994, Kondratyuk et al 1998, Marmor et al 2011). Die Ausbreitung von Strauchflechten wie den Moosbärten, der Sparrigen Pflaumenflechte und den Bartflechten erfolgt meist durch Bruchstücke und ist deshalb hauptsächlich lokal. Eine Ausbreitung über grössere Entfernung ist selten und trägt nur in ei- nem geringen Masse zur Ausbreitung dieser Artengruppe bei (Renhorn \& Esseen 1995). Diese Arten sind daher auf dauerhafte Lebensräume, wie sie autochthone Bestände darstellen, angewiesen und werden als Altwald-Flechten bezeichnet (Scheidegger \& Stofer 2015). Im Gegensatz dazu fanden Kruys \& Jonsson (1997), dass das Ausbreitungsvermögen von Stecknadelflechten gut entwickelt ist. Folglich kann ihre Beschränkung auf autochthone Bestände wahrscheinlich am besten durch das Vorhandensein geeigneter Mikrohabitate wie Borkenrisse an alten Bäumen erklärt werden (Tibell 1992). Solche Arten werden als Altbaum-Flechten bezeichnet (Scheidegger \& Stofer 2015).

Die Indikatorarten konnten auch, wenn auch deutlich seltener, in nicht autochthonen Waldbeständen beobachtet werden. Bei der Echten Lungenflechte, einer Flaggschiffart im Waldnaturschutz, stammten mit einem Anteil von 30\% vergleichsweise viele Nachweise aus Waldbeständen, die als nicht autochthon eingestuft worden waren. Solche Vor- 
kommen können den Charakter von Reliktvorkommen auf sehr alten Bäumen haben, die in einem autochthonen Bestand aufgewachsen sind und heute in einer Aufforstung stehen. Von der Echten Lungenflechte ist auch bekannt, dass sie auf Bäumen überleben kann, die während eines grossflächigen Waldbrandes als Einzelbäume überlebt haben (Wagner et al 2006, Werth et al 2006a, Werth et al 2006b).

Wie in Studien aus Deutschland, Italien und Rumänien (Ardelean et al 2016, Boch et al 2013, Nascimbene et al 2013) war auch bei uns die Anzahl untersuchter Flechtenarten in den nicht bewirtschafteten autochthonen Waldbeständen höher als in den bewirtschafteten autochthonen. Vorkommen einzelner Indikatorflechten können zwar herangezogen werden, um zwischen autochthonen und nicht autochthonen Gebirgswäldern zu unterscheiden, für die Unterscheidung zwischen bewirtschafteten und nicht bewirtschafteten Gebirgswaldbeständen eignen sich aber die meisten der hier untersuchten Arten nicht. Dies könnte daran liegen, dass die Schweizer Gebirgswälder seit Ende des 19. Jahrhunderts nicht mehr übernutzt werden und auch bereits seit vielen Jahren die waldbaulichen Eingriffe der Erhaltung der kleinräumigen Waldstruktur dienen, weshalb auf grössere Räumungen verzichtet wird (Brändli \& Imesch 2015). Nur zwei Indikatoren, die Echte Lungenflechte und die Gruppe der Weissfleckenflechten, waren mit autochthonen, nicht bewirtschafteten Waldbeständen assoziiert. Die Echte Lungenflechte ist aufgrund ihrer geringen Ausbreitungsfähigkeit als Indikator für Altwälder mit langfristiger Waldkontinuität bekannt (Öckinger et al 2005, Scheidegger \& Werth 2009, Werth et al 2006a). Es wird erwartet, dass eine hohe Anzahl weiterer Flechten, von denen viele in der Schweiz bedroht sind, ein ähnliches Muster aufweisen. Viele dieser Arten sind aber nur für Fachleute nach eingehender Einarbeitung im Gelände zu erkennen.

\section{Erkennen autochthoner Bestände anhand baumbewohnender Flechten}

Wir konnten einen signifikanten Einfluss der biogeografischen Regionen auf die getesteten Indikatorarten feststellen. Probeflächen im Jura und in den Südalpen wiesen deutlich weniger Indikatorarten auf als Flächen in den Nord- und Zentralalpen (Dymytrova et al 2018). Dies unterstreicht die Notwendigkeit, Indikatorarten für lokale und regionale Untersuchungen sorgfältig auszuwählen. Eine Auswahl an verlässlichen Indikatorarten für einzelne geografische Regionen sowie für die Schweizer Tieflagen konnte im Rahmen des LFI aber nicht geprüft werden.

Im Rahmen des LFI-Projektes hatte jedes Feldteam nur zwei Minuten Zeit pro Probefläche für die Flechtenerhebung. Diese zeitliche Einschränkung verringerte die Wahrscheinlichkeit, die potenziellen
Indikatorflechten zu erkennen, deutlich, zumal die Beobachtung von keinen Flechtenspezialisten durchgeführt wurde. In unserer Studie wurden aus zeitlichen Gründen im Schnitt nur drei Bäume innerhalb einer Probefläche untersucht. Laut den meisten europäischen Handbüchern zum Flechtenmonitoring (Asta et al 2002, Nimis et al 2002, Stofer et al 2012) sollten mindestens zehn Bäume innerhalb einer Probefläche untersucht werden. Um dies zu ermöglichen, sollte die Zeit für die Flechtenbeobachtung auf 15 bis 20 Minuten erhöht werden. In der Praxis wird der Fokus weniger auf kleinen Probeflächen als vielmehr auf der Ebene eines Bestandes liegen. Die Sensitivität der Methode kann verglichen mit der Ansprache auf LFI-Probeflächen deutlich verbessert werden, wenn die beurteilte Fläche auf eine Hektare erhöht wird.

\section{Schlussfolgerungen und Ausblick}

Basierend auf Daten des Landesforstinventars mit 3510 Gebirgswald-Probeflächen, zeigte unsere Studie eine starke Bindung von neun leicht erkennbaren Flechtenarten an autochthone Gebirgswälder der Schweiz. Da diese Arten jedoch nicht ausschliesslich in autochthonen Waldbeständen vorkamen, sollte das gleichzeitige Auftreten mehrerer Indikatorflechten berücksichtigt werden, um die Bestandesautochthonie angemessen zu kennzeichnen. Die Verwendung der neun Indikatorflechten empfehlen wir hauptsächlich Waldfachleuten als ein einfaches und effizientes Werkzeug, um im Gebirgswald autochthone Waldbestände als Vorranggebiete für die Erhaltung der Biodiversität zu erkennen.

Für lokale und regionale Untersuchungen sind die Indikatorarten sorgfältig auszuwählen. Zudem sind weitere Untersuchungen erforderlich, um verlässliche Indikatoren insbesondere für die Schweizer Tieflagenwälder zu erhalten. Autochthone Waldbestände im Tiefland zeichnen sich jedoch vor allem durch schwierig anzusprechende Krustenflechten aus, die nur durch Flechtenspezialisten bestimmt werden können.

Eingereicht: 2. April 2019, akzeptiert (mit Review): 13. Juli 2019

\section{Dank}

Wir danken den Feldteams der dritten LFI-Erhebung für die Datenerhebung, Christian Ginzler für seine Analysen zur Waldkontinuität und Rolf Meile für die Unterstützung bei der Abfrage der LFIDatenbank. Peter Brassel danken wir für die Unterstützung der Untersuchungen in einer frühen Projektphase und Volkmar Wirth für wertvolle Hinweise zu Indikatorflechten. Das Projekt wurde finanziert durch das Bundesamt für Umwelt und die Eidgenös- 
sische Forschungsanstalt für Wald, Schnee und Landschaft im Rahmen des gemeinsamen Programmes LFI.

\section{Literatur}

ARDELEAN IV, KELLER C, SCHEIDEGGER C (2016) Effects of management on lichen species richness, ecological traits and community structure in the Rodnei Mountains National Park (Romania). PLoS ONE 10 (12): e0145808.

ARSENAULT A, GOWARD T (2016) Macrolichen diversity as an indicator of stand age and ecosystem resilience along a precipitation gradient in humid forests of inland British Columbia, Canada. Ecol Indic 69: 730-738.

ASTA J, ERHARDT W, FERRETTI M, FORNASIER F, KIRSCHBAUM U, NIMIS PL ET AL (2002) European guideline for mapping lichen diversity as an indicator of environmental stress. Brussels: European Commission, Document 03-1-16. 20 p.

BAFU (2011) Liste der national prioritären Arten. Arten mit nationaler Priorität für die Erhaltung und Förderung. Stand 2010. Bern: Bundesamt Umwelt. $132 \mathrm{p}$.

BOCH S, PRATI D, HESSENMÖLLER D, SCHULZE ED, FISCHER M (2013) Richness of lichen species, especially of threatened ones, is promoted by management methods furthering stand continuity. PLoS One 8 (1): 1-9.

BRÄNDLI UB, IMESCH N (2015) Verjüngung. In: Rigling A, Schaffer HP, editors. Waldbericht 2015. Zustand und Nutzung des Schweizer Waldes. Bern: Bundesamt Umwelt. pp. 74-75.

BRIN A, VALLADARES L, LADET S, BOUGET C (2016) Effects of forest continuity on flying saproxylic beetle assemblages in small woodlots embedded in agricultural landscapes. Biodiv Conserv 25: 587-602.

CÁCERES MD (2013) How to use the indicspecies package (ver. 1.7.1). Solsona: Centre Tecnològic Forestal de Catalunya. 29 p. https://cran.r-project.org/web/packages/indicspecies/vignettes/ indicspeciesTutorial.pdf (9.7.2019).

CARO TM (2010) Conservation by Proxy: indicator, umbrella, keystone, flagship, and other surrogate species. Washington DC: Island Press. $374 \mathrm{p}$.

COPPINS AM, COPPINS BJ (2002) Indices of ecological continuity for woodland epiphytic lichen habitats in the British Isles. London: British Lichen Society. 36 p.

DUC P, BRÄNDLI UB, HEROLD BONARDI A, RÖSLER E, THÜRIG E ET AL (2010) Holzproduktion. In: Brändli UB, editor. Schweizerisches Landesforstinventar. Ergebnisse der dritten Erhebung 2004-2006. Birmensdorf: Eidgenössische Forsch.anstalt WSL. pp. 143-184.

DUFRÊNE M, LEGENDRE P (1997) Species assemblages and indicator species: the need for a flexible assymetrical approach. Ecol Monogr 67: 345-366.

DYMYTROVA L, BRÄNDLI UB, GINZLER C, SCHEIDEGGER C (2018) Forest history and epiphytic lichens: Testing indicators for assessing forest autochthony in Switzerland. Ecol Indic 84: 194-207.

ELLIS CJ (2012) Lichen epiphyte diversity: A species, community and trait-based review. Perspec Plant Ecol Evol Syst 14 (2): 131-152.

FREY E (1958) Die anthropogenen Einflüsse auf die Flechtenflora und -vegetation in verschiedenen Gebieten der Schweiz. Ein Beitrag zum Problem der Ausbreitung und Wanderung der Flechten. Veröff Geobot Inst Rübel Zür 33: 91-107.

FRITZ Ö, GUSTAFSSON L, LARSSON K (2008) Does forest continuity matter in conservation? A study of epiphytic lichens and bryophytes in beech forests of southern Sweden. Biol Conserv 141: 655-668.

GAUSLAA Y (1994) Lobaria pulmonaria, an indicator of species-rich forests of long ecological continuity. Blyttia 52: 119-128.
GAUSLAA Y, OHLSON M (1997) Continuity and epiphytic lichens in Norwegian forests. A historical perspective. Blyttia 55: 15-27.

GINZLER C, BRÄNDLI UB, HÄGELI M (2011) Waldflächenentwicklung der letzten 120 Jahre in der Schweiz. Schweiz Z Forstwes 162: 337-343. doi: 10.3188/szf.2011.0337

GRAAE BJ, SUNDE PB (2000) The impact of forest continuity and management on forest floor vegetation evaluated by species traits. Ecography 23: 720-731.

GRAHAM CH, MORITZ C, WILLIAMS SE (2006) Habitat history improves prediction of biodiversity in rainforest fauna. Proc Natl Acad Sci USA 103: 632-636.

IMESCH N, STADLER B, BOLLIGER M, SCHNEIDER O (2015) Biodiversität im Wald: Ziele und Massnahmen. Vollzugshilfe zur Erhaltung und Förderung der biologischen Vielfalt im Schweizer Wald. Bern: Bundesamt Umwelt. $186 \mathrm{p}$.

JOSEFSSON T, HELLBERG E, ÖSTLUND L (2005) Influence of habitat history on the distribution of Usnea longissima in boreal Scandinavia: a methodological case study. Lichenologist 37: 555-567.

KELLER M, EDITOR (2011) Swiss National Forest Inventory. Manual of fied survey 2004-2007. Birmensdorf: Eidg. Forsch.anstalt WSL. $271 \mathrm{p}$

KONDRATYUK SY, COPPINS BJ, ZELENKO SD, KHODOSOVTSEV AY, COPPINS AM ET AL (1998) Lobarion lichens as indicators of primeval forests in the Ukrainian part of the proposed trilateral reserve "Eastern Carpathians". In: Kondratyuk SY, Coppins BJ, editors. Lobarion lichens as indicators of the primeval forests of the Eastern Carpathians. Kiev: M. H. Kholodny Institute of Botany. pp. 64-79.

KRUYS N, JONSSON B (1997) Insular patterns of calicioid lichens in a boreal old-growth forest-wetland mosaic. Ecography 20: 605-613

LANZ A, ABEGG M, BRÄNDLI UB, CAMIN P, CIOLDI F ET AL (2016) Switzerland. In: Vidal C, Alberdi IA, Hernández Mateo L, Redmond JJ, editors. National Forest Inventories: Assessment of wood availability and use. Cham: Springer International Publishing. pp. 783-805.

MARMOR L, TÕRRA T, SAAG L, RANDLANE T (2011) Effects of forest continuity and tree age on epiphytic lichen biota in coniferous forests in Estonia. Ecol Indic 11: 1270-1276.

MCCUNE B (2000) Lichen communities as indicators of forest health. Bryologist 103: 353-356.

NASCIMBENE J, THOR G, NIMIS PL (2013) Effects of forest management on epiphytic lichens in temperate deciduous forests of Europe: a review. For Ecol Manage 298: 27-38.

NIMIS PL, SCHEIDEGGER C, WOLSELEY PA (2002) Monitoring with lichens, Monitoring lichens. Dordrecht: Kluwer. 408 p.

ÖCKINGER E, NIKLASSON M, NILSSON SG (2005) Is local distribution of the epiphytic lichen Lobaria pulmonaria limited by dispersal capacity or habitat quality? Biodiv Conserv 14: 759-773.

RENHORN KE, ESSEEN PA (1995) Biomass growth in five alectorioid lichen epiphytes. In: Scheidegger C, Wolseley PA, Thor G, editors. Conservation biology of lichenised fungi. Birmensdorf: Eidgenöss Forsch.anstalt WSL, Mitt 70 (1). pp. 133-140.

ROSE F (1976) Lichenological indicators of age and environmental continuity in woodlands. In: Brown DH, Hawksworth DL, Bailey $\mathrm{RH}$, editors. Lichenology: progress and problems. London: Academic Press. pp. 279-307.

SCHEIDEGGER C, STOFER S (2003) Identifizierung von autochthonen Beständen - Feldunterlagen und Erkennungstabellen leicht kenntlicher Indikatorflechten für die dritte LFI-Erhebung. Birmensdorf: Eidgenöss Forsch.anstalt WSL. 23 p.

SCHEIDEGGER C, STOFER S (2015) Bedeutung alter Wälder für Flechten: Schlüsselstrukturen, Vernetzung, ökologische Kontinuität. Schweiz Z Forstwes 166: 75-82. doi: 10.3188/szf.2015.0075

SCHEIDEGGER C, WERTH S (2009) Conservation strategies for lichens: insights from population biology. Fungal Biol Rev 23: 55-66. 
STOFER S, CALATAYUD V, GIORDANI P, NEVILLE P (2012) Assessment of epiphytic lichen diversity. In: ICP Forests, editor. Manual on methods and criteria for harmonized sampling, assessment, monitoring and analysis of the effects of air pollution on forests. Hamburg: ICP Forests Programme Co-ordinating Centre. pp. 1-14.

TIBELL L (1992) Crustose lichens as indicators of forest continuity in boreal coniferous forests. Nordic J Bot. 12: 427-450.

WAGNER HH, WERTH S, KALWIJ JM, BOLLI JC, SCHEIDEGGER C (2006) Modelling forest recolonization by an epiphytic lichen using a landscape genetic approach. Landsc Ecol 21: 849-865.

WERTH S, WAGNER HH, GUGERLI F, HOLDEREGGER R, CSENCSICS D ET AL (2006A) Quantifying dispersal and establishment limitation in a population of an epiphytic lichen. Ecology 87: 2037-2046.

WERTH S, WAGNER HH, HOLDEREGGER R, KALWIJ JM, SCHEIDEG GER C (2006B) Effect of disturbances on the genetic diversity of an old-forest associated lichen. Mol Ecol 15: 911-921.

\section{Reconnaître les forêts de montagne autochtones de Suisse par l'intermédiaire de lichens épiphytes}

Les vieux peuplements forestiers non perturbés ayant une longue histoire forestière présentent un intérêt particulier pour la conservation de la nature et des espèces. Les lichens épiphytes sont souvent utilisés comme indicateurs de ces peuplements autochtones. Dans cette étude, nous avons testé dans quelle mesure 14 espèces de lichens épiphytes, qui sont faciles à reconnaître pour les praticiens forestiers et qui ont un faible potentiel de dispersion, des exigences de substrat spécifiques et sont souvent limitées aux arbres âgés, seraient des espèces indicatrices appropriées pour les peuplements forestiers autochtones dans les forêts suisses des montagnes. En tant qu'autochtones, nous avons considéré les peuplements forestiers avec une teneur en résineux proche de la nature, un âge minimum de 90 ans, un couvert forestier continu de plus de 120 ans et aucune trace $d^{\prime}$ intervention historique telle que la coupe rase ou le boisement. Dans le cadre de l'étude, les équipes de terrain régulières de I'Inventaire forestier national suisse (IFN) ont inclus les espèces indicatrices potentielles dans la troisième enquête (2004-2006) sur toutes les placettes échantillonnées du réseau de $1.4 \mathrm{~km}$ sur environ trois arbres chacune. Les données pour les 3510 placettes de la forêt de montagne ont ensuite été évaluées à l'aide des données de I'IFN sur la structure, I'histoire et la gestion de la forêt. Dans l'ensemble, le nombre d'espèces indicatrices était significativement plus élevé dans les peuplements forestiers autochtones que dans les peuplements non autochtones, bien qu'il varie considérablement selon l'altitude et la région biogéographique. Nos recherches ont montré que les lichens fruticuleux du genre Bryoria sp. div. et Usnea sp. div., Evernia divaricata, Letharia vulpina, les lichens foliacés Cetrelia olivetorum, Lobaria pulmonaria ainsi que les lichens crustacés Lecanactis abietina et Microcalicium disseminatum, Calicium sp. div., Chaenotheca sp. div. et Chrysothrix candelaris sont des indicateurs fiables des peuplements forestiers autochtones des forêts de montagne suisses, surtout si plusieurs espèces indicatrices sont présentes ensemble. Les lichens testés conviennent donc dans la pratique pour l'identification simple des peuplements forestiers autochtones, par exemple pour la délimitation des réserves forestières ou des îlots de sénescence. Ces peuplements sont d'une grande importance pour la promotion de la biodiversité dans un paysage forestier géré depuis des siècles.
WIRTH V (1976) Veränderungen der Flechtenflora und Flechtenvegetation in der Bundesrepublik Deutschland. Schr.reihe Veg. kd. 10: 177-203.

WIRTH V (2002) Indikator Flechte. Naturschutz aus der Flechtenperspektive. Stuttg Beitr Nat.kd, Serie C 50: 1-96.

\section{Quellen}

BRÄNDLI UB, CIODLI F, FISCHER C, HUBER M (2015) Schweizerisches Landesforstinventar - Spezialauswertungen zum Waldbericht 2015 im Internet. Birmensdorf: Eidgenöss Forsch.anstalt WSL. www.Ifi.ch/publ/waldbericht/2015.php (9.7.2019)

\section{Recognizing autochthonous mountain forests in Switzerland by means of epiphytic lichens}

Old undisturbed forest stands with a long forest history are of particular interest for nature and species conservation. Epiphytic lichens are often used as indicators for such autochthonous stands. In this study, we tested to what extent 14 taxa of epiphytic lichens, which are easy to identify by forest practitioners and have a low dispersal potential, have specific requirements for micro habitats and are often dependent on old trees, would be suitable indicator species for autochthonous forest stands in the Swiss mountain forests. As autochthonous, we considered forest stands with a near-natural conifer composition, a minimum age of 90 years, a continuous forest cover over 120 years and no evidence of historical intervention such as clear cutting or afforestation. For the study, the regular field teams of the Swiss National Forest Inventory (NFI) included the potential indicator species in the third survey (2004-2006) on all plots in the $1.4 \mathrm{~km}$ network on about three trees each. The data for the 3510 plots in the mountain forest were then evaluated using the NFI data on forest structure, forest history and forest management. Overall, the number of indicator species was significantly higher in autochthonous forest stands than in non-autochthonous ones, although it varied considerably with altitude and biogeographical region. Our investigations showed that fruticose lichens of the genera Bryoria and Usnea, Evernia divaricata and Letharia vulpina, foliose lichens such as Cetrelia olivetorum and Lobaria pulmonaria, and crustose lichens such as Lecanactis abietina and Microcalicium disseminatum, Calicium sp. div. and Chaenotheca sp. div. as well as Chrysothrix candelaris are reliable indicators for autochthonous stands in Swiss mountain forests, especially if several indicator species occur together. The lichens tested are therefore suitable in practice for a simple identification of autochthonous forest stands, e.g. for delimiting forest reserves or old forest patches. These stands are of high importance for the promotion of biodiversity in a forest landscape that has been managed for centuries. 